\title{
Endobronchial Ultrasound Bronchoscopy to the Heart of the Matter
}

\author{
Anastasios F. Palamidas ${ }^{a}$ b Ulrich Rosendahlc Pallav L. Shah ${ }^{a, b}$ \\ ${ }^{a}$ NIHR Respiratory Biomedical Research Unit, Royal Brompton and Harefield NHS Foundation Trust and \\ Imperial College, ${ }^{b}$ Chelsea and Westminster Hospital, and ${ }^{\mathrm{C}}$ Department of Cardiac Surgery, Royal Brompton \\ Hospital, London, UK
}

\section{Established Facts}

- Angiosarcomas are rare tumours of the heart and great vessels that are usually underdiagnosed until they become symptomatic.

- Symptomatic angiosarcomas mark disease progression with a very small range of therapeutic options.

- Despite the need for early diagnosis, the surgical biopsy is usually delayed due to severe possible complications.

\section{Novel Insights}

- The convex probe endobronchial ultrasound technique with guided transbronchial needle aspiration utilities has been extended to recognise pulmonary emboli and obtain tissue diagnosis from cardiac masses.

- The transvascular biopsy procedure becomes more feasible and has fewer complications compared to surgery, depending on the bronchoscopist's experience.

\section{Key Words}

Endobronchial ultrasound · Bronchoscopy · Convex probe endobronchial ultrasound · Angiosarcoma - Transbronchial needle aspiration

\begin{abstract}
Endobronchial ultrasound has been one of the success stories of the last decade, and the utility of the procedure continues to expand. Originally, it was developed for the staging and diagnosis of lung cancer, but its use rapidly expanded to other malignancies and even benign disease. We present the case of a patient originally referred with suspected endocar-
\end{abstract}

ditis who was found to have a mass involving the right ventricle and the pulmonary outflow tract. Endobronchial ultrasound-guided biopsy was used to obtain a tissue diagnosis from the cardiac mass.

(c) 2016 S. Karger AG, Basel

\section{KARGER}

E-Mail karger@karger.com www.karger.com/res
C 2016 S. Karger AG, Basel

0025-7931/16/0922-0127\$39.50/0

\section{Introduction}

Endobronchial ultrasound (EBUS) mediastinoscopy by convex probe (CP)-EBUS is the method of choice for obtaining tissue samples from thoracic tumours adjacent to the trachea and bronchial airways or from mediastinal 
Fig. 1. Computed tomography of the chest. Mass from the right ventricle and the pulmonary tract extending into both pulmonary arteries (left and right panels). The tumour, located in the left main bronchus just below the carina, was made visible by the CP-EBUS.
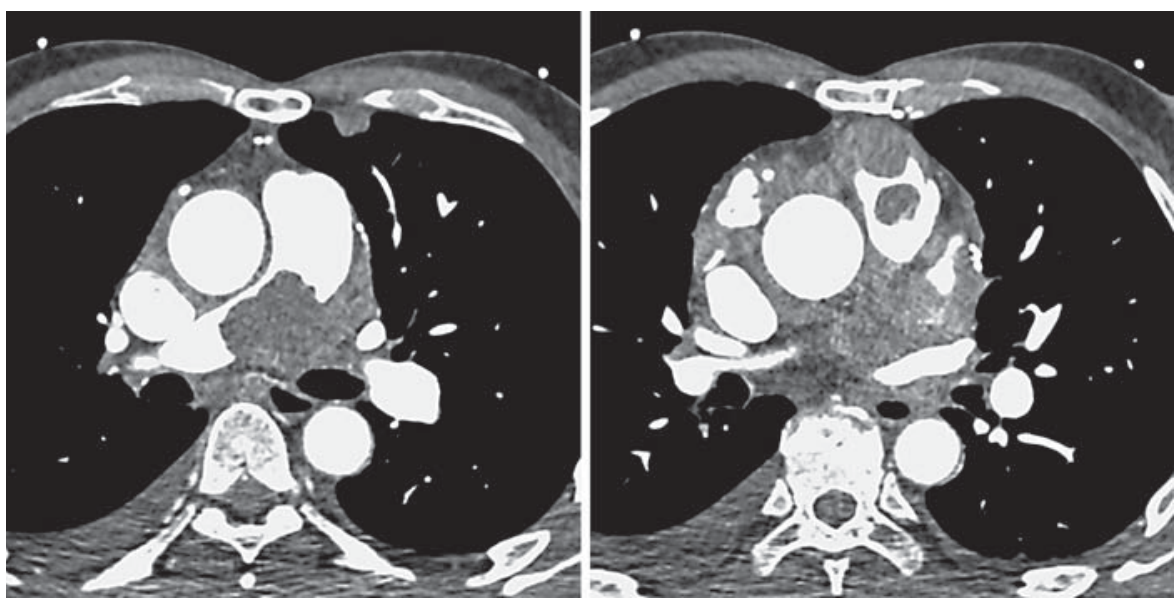

nodes when the disease is locally advanced [1]. Highquality randomised controlled trials have established the role of EBUS in the diagnosis of both malignant and benign disease $[2,3]$. Tissue acquisition for accurate diagnosis and staging is essential both for prognosis and determining therapeutic strategies [4]. The procedure is considered very specific and sensitive and has a low morbidity, which is considered ideal for an initial investigation [4]. The utility of EBUS has been further enhanced with improvement in molecular techniques, whereby analysis is possible on cytological samples [5]. The indications of EBUS continue to expand, and we present the case of a patient with a cardiac mass that was evaluated with EBUS.

\section{Case Report}

We report the case of a 69-year-old patient admitted as an urgent inpatient transfer with the diagnosis of endocarditis. He was symptomatic with fever and shortness of breath that was limiting his physical exertion. The patient was confined to bed or chair for more than $50 \%$ of his waking hours. His past medical history included left anterior descending coronary artery double bypass with internal mammary artery graft. He had chronic atrial fibrillation with risk factors including hypertension and hypercholesterolemia.

The patient was first assessed at our centre with a transthoracic echocardiogram which demonstrated an impaired ejection fraction $(20-30 \%)$. The right ventricle cavity volume was highly reduced and showed a severely impaired systolic function. The right atrium was severely dilated. The tricuspid valve was mildly thickened and retracted with incompetent leaflets. The Doppler colour flows also showed free low-pressure regurgitation. The pulmonary artery systolic pressure was estimated at about $34 \mathrm{~mm} \mathrm{Hg}$. The main findings though were multiple large and small echogenic and echolucent masses within the cavity of the right ventricle, mainly located at the outflow tract extending to the main pulmonary ar- tery and the right and left pulmonary arteries. Few mobile small lesions were also visible inside the right ventricular cavity. A mobile mass was also seen attached on the septal leaflet of the tricuspid valve. Masses were also found surrounding the pulmonary valve.

Computed tomography pulmonary angiography revealed that the right ventricular cavity was occupied by a large lobulated necrotic mass (fig. 1). A small tissue nodule was extending into the right atrial septum. The dilatation of the right atrium stretched out to the inferior vena cava and the hepatic veins, presumably reflecting a right heart obstruction pattern. The mass was found to extend to the right ventricle outflow tract and the main pulmonary artery, causing severe stenosis of both branching pulmonary arteries. No mediastinal adenopathy was detected, but small bilateral pleural effusions were evident. There were no other distal abnormalities.

Cardiovascular magnetic resonance imaging confirmed that there were several intracardiac masses (right ventricular base, septal wall, free wall, outflow tract) (fig. 2). A big mass $(33 \times 42 \mathrm{~mm}$ ) was also evident in the pulmonary bifurcation with extension to the adjacent mediastinum, causing significant obstruction of the main pulmonary artery and occlusion of the right pulmonary artery.

Tissue acquisition posed significant challenges due to its location and the clinical status of the patient. EBUS was determined to be the safest initial option (fig. 3). Topical anaesthesia with lignocaine $2 \%$ and mild sedation with $2 \mathrm{mg}$ of midazolam and $200 \mu \mathrm{g}$ of alfentanyl were applied. On bronchoscopy, no mediastinal or hilar nodes were detected. When the scope was positioned at the left tracheal margin at the level of the carina and below, the mass was visible, infiltrating the pulmonary trunk and the left main pulmonary artery. Further infiltration of the right ventricle was visible in the medial and anterior aspect of the left main bronchus. Biopsies from the cardiac mass were obtained with a 22 -gauge needle (Olympus Medical). The procedure was very well tolerated, and adequate histological core samples and cytology were obtained. No complications were observed after the procedure. Histology showed malignant tumour cells of epithelioid origin. Immunohistochemistry of the tumour cells was negative for TTF-1, keratin, and epithelial markers (MNF, CK5/6, CK7, CKAE1/3). The stain for vimentin was strongly positive. The pathology was consistent with a pleomorphic undifferentiated sarcoma. 

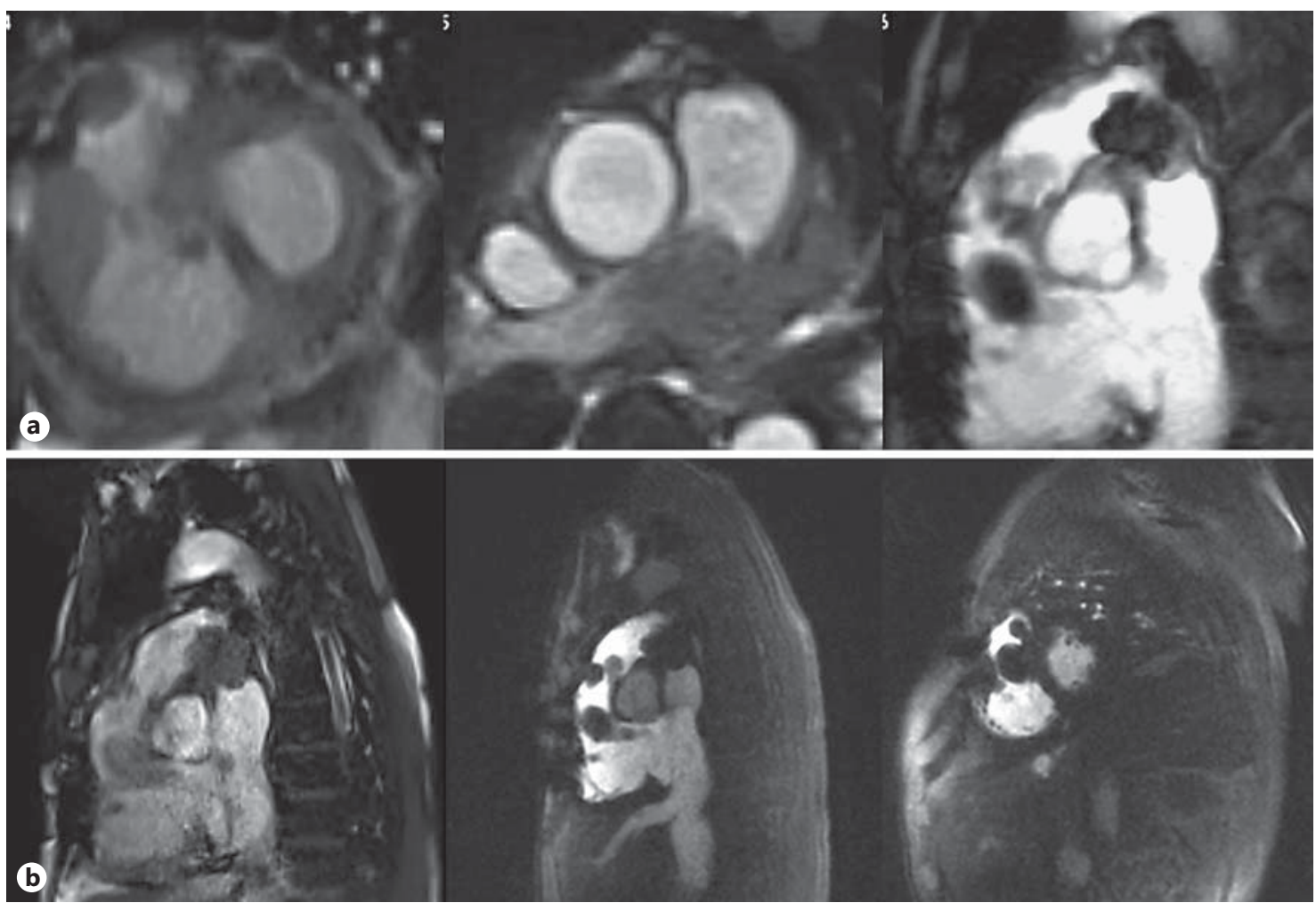

Fig. 2. Cardiovascular magnetic resonance images. a Transverse sections. b Sagittal sections confirming multiple masses in the right ventricle outflow and the pulmonary tract.
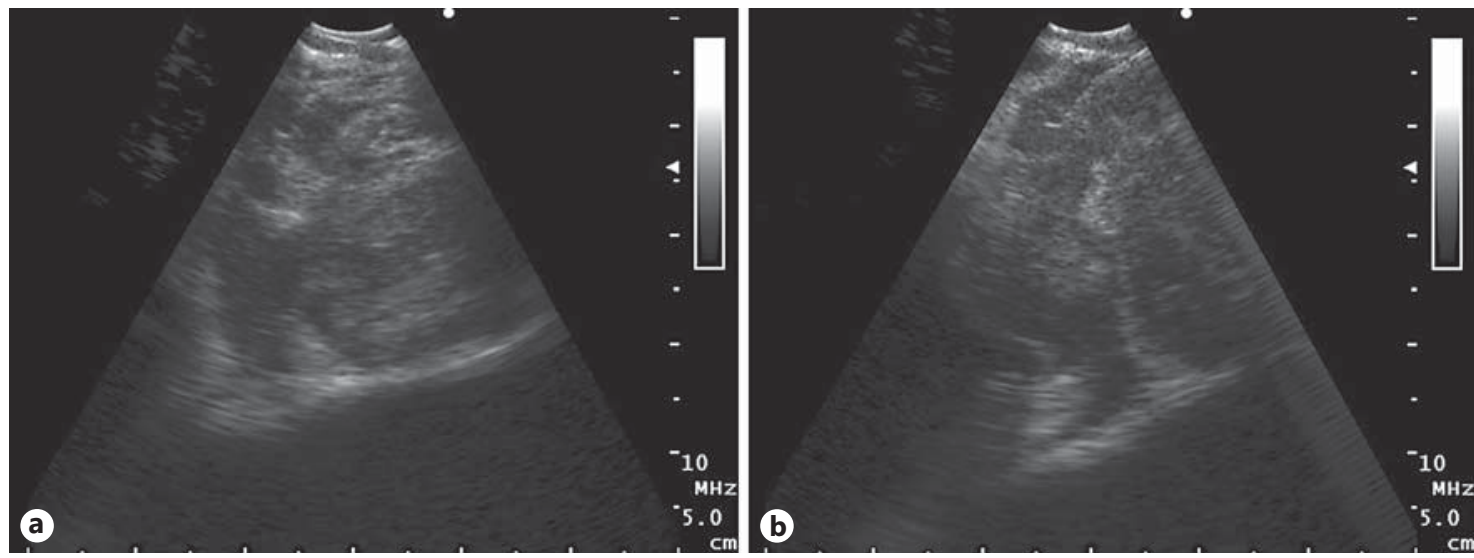

Fig. 3. CP-EBUS images. a The lesion of the great vessel in respect to the heart is shown. $\mathbf{b}$ Tissue acquisition from the lesion in the vessel.

\section{Discussion}

Primary neoplastic lesions of the heart and the great vessels are rare and usually benign (75\%). In adult patients, sarcomas are the commonest malignancies originating from the heart and the vessels surrounding it. The most frequent subtype is the pleomorphic undifferentiated sarcoma which, after the newest WHO classification, includes malignant fibrous histiocytoma [6]. Sarcomas originating from the great vessels are typically located in the pulmonary arteries. Venous sarcomas usually originate from the inferior vena cava and are not as frequent 
as their arterial counterpart. The prognosis is often discouraging because, at the time of referral, they have already metastasised or locally advanced [7].

Usually, the primary cardiac or vascular mediastinal tumours are misdiagnosed as thromboembolic disease or endocarditis. Pulmonary hypertension and obstructive heart failure symptoms and signs are also present. The diagnosis requires a high level of suspicion and surveillance and generally would be considered after the failure of antibiotics and anticoagulation therapy [7].

Accessing the thoracic vasculature and transbronchial left atrial catheterisation has been primarily attempted almost 50 years ago and is still performed with relative success in terms of diagnostic accuracy and low morbidity rates [8-10]. Despite the good results until the introduction and standardisation of the transbronchial needle aspiration (TBNA) procedure by Wang and Terry [11], these procedures were abandoned.

The EBUS technique with the integrated linear array probe into the bronchoscope has enabled real-time assistance-guided TBNA [12]. This report illustrates how the role of EBUS has evolved beyond the diagnosis and staging of lung cancer. With the use of the Doppler mode, vascular diseases and lesions can be recognised and approached more safely and easily.

Surgical excision remains the gold therapeutic standard but usually cannot be achieved with clear margins because the disease tends to extend to distal small pulmo- nary vessels with subclinical cancer thromboemboli. Combined therapy with neoadjuvant chemotherapy prior to surgery usually promises a better overall survival. Radiotherapy can also be used with caution due to the sensitivity of the cardiac tissues to radiation $[6,7]$. Diagnosis with EBUS has the advantage of facilitating neoadjuvant therapy and hence reserving surgery for therapeutic purposes as the final step in multimodality treatment. Cardiac transplantation is another feasible therapeutic modality, but this approach would be disappointing due to local recurrence or metastases [13].

In our case, due to the clinical condition of the patient and the extent of the disease, a tissue biopsy was attempted with EBUS as it was considered to be a less interventional procedure. Although transvascular biopsies have been reported before [7], and despite the fact that EBUS TBNA appears to be minimally invasive and feasible, their relative merits remain unclear. Both techniques are vulnerable to potential complications like haemorrhage, haematoma, haemomediastinum, infection mediastinitis, and pneumomediastinum. Furthermore, the physiology is more akin to a massive pulmonary embolism with hemodynamic instability and hypoxia. Due to the lack of sufficient clinical evidence to assess safety and the actual diagnostic yield, the decision for a transvascular or EBUS TBNA approach should be pragmatic. Each individual case should be considered after assessing the benefits and the risks for the individual patient.

\section{References}

1 De Leyn P, Dooms C, Kuzdzal J, et al: Revised ESTS guidelines for preoperative mediastinal lymph node staging for non-small cell lung cancer. Eur J Cardiothorac Surg 2014;45:787798.

2 Annema JT, van Meerbeeck JP, Rintoul RC, Dooms C, Deschepper E, Dekkers OM, De Leyn P, Braun J, Carroll NR, Praet M, de Ryck F, Vansteenkiste J, Vermassen F, Versteegh MI, Veseliç M, Nicholson AG, Rabe KF, Tournoy KG: Mediastinoscopy vs endosonography for mediastinal nodal staging of lung cancer: a randomized trial. JAMA 2010; 304:2245-2252.

- 3 Von Bartheld MB, Dekkers OM, Szlubowski $A$, et al: Endosonography versus conventional bronchoscopy for the diagnosis of sarcoidosis: the GRANULOMA randomized clinical trial. JAMA 2013;309:2457-2464.

4 Adams K, Shah PL, Edmonds L, Lim E: Test performance of endobronchial ultrasound and transbronchial needle aspiration biopsy for mediastinal staging in patients with lung cancer: systematic review and meta-analysis. Thorax 2009;64:757-762.

5 Nicholson AG, Gonzales D, Shah P, et al: Refining the diagnosis and EGFR status of nonsmall cell carcinoma in biopsy and cytologic material, using a panel of mucin staining, TTF-1, cytokeratin 5/6 and P63, and mutation analysis. J Thorac Oncol 2010;5:436441.

6 Mayer F, Aebert H, Rudert M, et al: Primary malignant sarcomas of the heart and great vessels in adult patients - a single centre experience. Oncologist 2007;12:1134-1142.

$\rightarrow$ Burke AP, Virmani R: Sarcomas of the great vessels. A clinicopathologic study. Cancer 1993;71:1761-1773.

$\checkmark 8$ Crymes TP, Fish RG, Smith DE: Complications of transbronchial left atrial puncture. Am Heart J 1959;58:46-52.

-9 Horowitz JC, Kleaveland K, Arenberg D: Endobronchial biopsy of an intrapulmonary ar- terial mass. J Bronchology Interv Pulmonol 2013;20:93-95.

10 Dusemund F, Schneider T, Zeisel C, Rothermundt C, Kluckert T, Schmid S, Brutsche $\mathrm{MH}$ : Endobronchial ultrasound-guided transbronchial needle aspiration of an intravascular sarcoma metastasis. Respiration 2013;86:430-432.

11 Wang KP, Terry PB: Transbronchial needle aspiration in the diagnosis and staging of bronchogenic carcinoma. Am Rev Respir Dis 1983; 127:344-347.

12 Herth FJ, Eberhardt R, Vilmann P, Krasnik M, Ernst A: Real-time endobronchial ultrasound guided transbronchial needle aspiration for sampling mediastinal lymph nodes. Thorax 2006;61:795-798.

$\checkmark 13$ Talbot SM, Taub RN, Keohan ML, Edwards N, Galantowicz ME, Schulman LL: Combined heart and lung transplantation for unresectable primary cardiac sarcoma. J Thorac Cardiovasc Surg 2002;124:1145-1148. 